\title{
Integrating Renewable Energy Resources into the Smart Grid: Recent Developments in Information and Communication Technologies
}

\author{
Mubashir Husain Rehmani, Martin Reisslein, Abderrezak Rachedi, Melike Erol-Kantarci, and Milena Radenkovic
}

\begin{abstract}
Rising energy costs, losses in the present-day electricity grid, risks from nuclear power generation, and global environmental changes are motivating a transformation of the conventional ways of generating electricity. Globally, there is a desire to rely more on renewable energy resources (RERs) for electricity generation. RERs reduce green house gas emissions and may have economic benefits, e.g., through applying demand side management with dynamic pricing so as to shift loads from fossil fuel-based generators to RERs. The electricity grid is presently evolving towards an intelligent grid, the so-called smart grid (SG). One of the major goals of the future SG is to move towards $100 \%$ electricity generation from RERs, i.e., towards a $100 \%$ renewable grid. However, the disparate, intermittent, and typically widely geographically distributed nature of RERs complicates the integration of RERs into the SG. Moreover, individual RERs have generally lower capacity than conventional fossil-fuel plants, and these RERs are based on a wide spectrum of different technologies. In this article, we give an overview of recent efforts that aim to integrate RERs into the SG. We outline the integration of RERs into the SG along with their supporting communication networks. We also discuss ongoing projects that seek to integrate RERs into the SG around the globe. Finally, we outline future research directions on integrating RERs into the SG.
\end{abstract}

Index Terms-Renewable energy resources (RERs), Distributed energy resources (DERs), Advanced metering infrastructure (AMI), Communication architecture, Smart grid (SG).

\section{INTRODUCTION}

\section{A. Motivation: Need for Integration of Renewable Energy Resources with Smart Grid}

Nowadays, there is a high demand for renewable energy and this demand is increasing due to rising energy cost and global environmental changes. The existing power grid relies heavily on conventional fossil fuel-based electricity generation units. Moving electrical energy from these generation units

Please direct correspondence to Mubashir Husain Rehmani.

M.H. Rehmani is with the Telecom. Softw. \& Sys. Group, Waterford Inst. of Techn., Carriganore, Waterford, X91 P20H, Ireland (email: mshrehmani@gmail.com) Phone: +353 (0)830450591.

M. Reisslein is with Electrical, Computer and Energy Eng., Arizona State University, Tempe, AZ 85287-5706 USA (e-mail: reisslein@asu.edu).

A. Rachedi is with Gaspard Monge CS Lab., Univ. Paris-Est, 75420 Champs sur Marne, France. (e-mail: rachedi@u-pem.fr).

M.E. Kantarci is with Electrical Eng. \& Computer Science, Univ. of Ottawa, ON, Canada. (email: melike.erolkantarci@uottawa.ca).

M. Radenkovic is with Computer Science \& IT, Wollaton Rd., Univ. of Nottingham, Nottingham, NG8 1BB, UK (email: mvr@cs.nott.ac.uk). over the distribution lines to the customers in the presentday electricity grid incurs significant power losses. In addition, many conventional fossil fuel-based electricity generation units pose hazards, e.g., hazards from nuclear power plants. Thus, there is a global push to reform the electricity generation by moving away from fossil-fuel based plants and moving towards RERs. By using RERs, $\mathrm{CO}_{2}$ emission can be globally reduced. In addition, RERs can enable economic benefits, both for customers and utilities, by exploiting demand side management (DSM) to dynamically adjust energy pricing so as to judiciously share the load between fossil fuel-based plants and RERs.

The current electricity grid is evolving into an intelligent grid, widely referred to as smart grid (SG) [1]. In the future $\mathrm{SG}$, one major goal is to move towards achieving a $100 \%$ renewable grid i.e., to make the future grid an inverterdominated grid [2]. An inverter is an electronic device which connects RERs to the power grid [2]. However, an inverterdominated grid poses several challenges. First, RERs are typically intermittent in nature, e.g., available only during certain hours of the day or seasons of the year. Second, RERs are based on highly heterogeneous technologies that each have their unique characteristics, e.g., solar plants have very different characteristics compared to wind farms or tidal wave power plants. Third, RERs have typically less capacity compared to their fossil fuel-based counterparts. Finally, RERs are more spread out in terms of their geographic locations [3]. These unique properties of inverter-connected RERs make the integration into the SG very challenging.

The SG has two types of flows: two-way energy flows and two-way information flows [3], [4]. The concept of two-way energy flows means that energy can be generated by power generators and delivered to the customers and/or energy can be generated by the customers and be injected back into the power grid. The concept of two-way information flows means that utilities have access to real-time information and at the same time customers control dynamic energy flows and collect various power related parameters. Energy flows transport electrical energy to meet the customer electricity demands. Information flows transport information (data) for monitoring the status and collecting various types of information in the SG as well as for controlling the dynamic energy flows. An efficient communication system, i.e., fast reliable information flows, supports good system observability and controllability, which will ultimately result in a secure, 


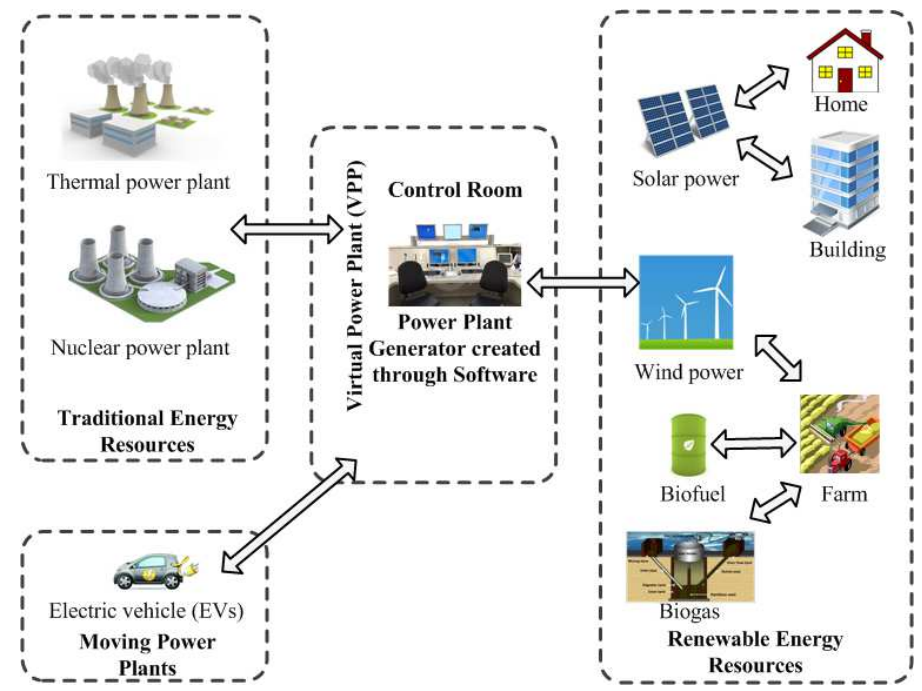

Fig. 1. Illustration of a typical virtual power plant (VPP), which combines different energy resources through centralized software [5].

reliable, and safe SG. Efficient energy flows will avoid load shedding, i.e., situations where the grid is not able to meet the electricity demands. Two-way communication is necessary in the SG to support these two types of flows. In fact, this two-way communication is an integral part of the future SG to accommodate RERs. In summary, communication systems supporting two-way communication will play a vital role in the integration of RERs into the SG.

In this article, we present first more comprehensive overview of recent developments that have strived to integrate RERs into the SG. We first provide an overview of RERs and the SG along with a discussion on the entity that "stitches" SG resources together, namely the "virtual power plant". Then, we provide an in-depth discussion on the integration of RERs with the SG and communication networks, covering cellular networks, rail transport system, and data centers. We also highlight on-going projects on this RERs integration into the SG around the globe. Finally, we outline future research directions on integrating RERs into the SG.

\section{B. Comparison with Related Survey Articles}

Extensive prior work has examined the integration of distributed energy resources (DERs) and renewable energy resources (RERs) into the SG. Several special journal issues have been published on the integration of RERs into the SG, see for instance, [6], [7]. Also, some books have been published on this topic [8]-[10]. These prior publications examined the integration of RERs into the SG from different perspectives. For instance, communication systems for grid integration have been discussed in [3]. A road map to integration has been outlined in [11]. The concept of the virtual power plant (VPP) and the integration of RERs into VPPs has been introduced in [5]. Recently, key technologies for the integration of multiple types of RERs have been discussed in [12]. To be more precise, the study [12] focused on the multi-time-frame robust scheduling/dispatch problem. The integration of renewable distributed generators into the SG has been considered in [13].
Complementary to these prior articles, we provide an up-todate overview of the communication aspects arising from the integration of RERs into the SG.

\section{Article Structure}

The remainder of this article is organized as follows: In Section II, we provide an overview of RERs and the SG. In Section III, we survey studies on the integration of RERs into the SG and the communication networks supporting the SG information flows. We outline future research directions on integrating RERs into the SG in Section IV and finally, we conclude the article in Section V.

\section{Renewable Energy Resources And the SMART GRID: AN OVERVIEW}

\section{A. Smart Grid}

The smart grid (SG) is the next generation electric grid which merges information and communication technologies (ICT) and control systems with the power grid. The SG becomes "smart" when it fully supports two-way information (communication) flows and two-way energy flows that are efficiently controlled based on real-time information [3], [4]. An SG consists typically of home area networks (HANs), neighborhood area networks (NANs), and a wide area network (WAN). A HAN is a local SG network segment that interconnects distributed renewable energy generators (e.g., solar panels) and electricity consuming devices in a home. HANs support the charging of electric vehicles (EVs) and account for a large portion of the overall electricity consumption; it is estimated that over $50 \%$ of the electricity is consumed in homes [4]. The NAN gathers information from multiple HANs and relays this information to the WAN. The WAN serves as a communication backbone to connect sub-stations, DERs, data aggregation points, network gateways, and utility data centers. In addition to HANs, NANs, and the WAN, the SG also contains thousands of smart meters that are located at user premises, forming the advanced metering infrastructure (AMI).

\section{B. Renewable Energy Resources (RERs)}

RERs, such as light, vibration, biogas, biofuel, heat, radio frequency $(\mathrm{RF})$, and wind, are abundant energy resources in the natural environment around us. Renewable energy can be gained from light which can be in the form of sunlight (e.g., large scale solar farms) or artificial light (e.g., indoor environments in homes or office buildings). Photovoltaic cells are normally used to obtain solar energy, however, solar energy is seasonal and its availability varies with the geographic location. Wind is another form of renewable energy in which linear air motion is used by wind turbines in wind farms to generate electricity. Similar to solar energy, wind energy also depends on the climate and geographic location. Vibration, heat (e.g., arising from chemical reactions), and RF are forms of renewable energy that are typically not integrated back into the power grid due to their small scale generation capacity. One common perception is that due to the intermittent and 
disparate nature of RERs, a complete reliance on RERs is not feasible; until sufficient RER based energy storage capacities become available [14]. Another perception is that RERs have numerous advantages; thus utilities should carefully plan and move toward achieving a $100 \%$ renewable grid, i.e., an inverter-dominated grid.

\section{Virtual Power Plant: The Stitching Entity of SG Resources}

One of the core questions is how to manage the conventional energy resources, diversified distributed RERs, and moving small power plants, e.g., in the form of EVs that feed back into the grid, in the future SG? How can these resources be effectively stitched together and how can these different entities in the SG be efficiently coordinated? One promising answer is through the concept of the virtual power plant (VPP) [5]. We define a VPP as a central virtual entity that combines, manages, and controls geographically distributed energy resources. These energy resources generate electricity and sell the generated power (that is not used by the resource itself) to the main electricity grid [5], [15]. Fig. 1 shows the concept of the VPP which is basically a power plant generator created through software. In fact, the VPP is a virtual entity and does not have any physical infrastructure, e.g., no physical turbines. The VPP acts as central control entity that combines traditional energy resources, moving power plants (EVs), and RERs.

VPPs have several advantages [15]. First, RERs can be stitched together for short periods of time. Second, VPPs enable small energy producers, such as homes, small commercial buildings, and industries, to participate in energy markets. Small energy producers can use the concept of net metering. In net metering, credit is provided to the customers when they inject surplus renewable energy into the main grid. The capacity of the VPP consists of RERs, DERs, and energy saved at homes through demand side management (DSM) during peak hours, as well as traditional energy resources. The grid performance can also be enhanced through EVs by controlling them through VPPs [4].

An alternative to the fine-grained control through the VPP concept is the traditional way to meet the power demands by increasing the production at power plants. When the power plants are unable to meet the peak demand, then load shedding or complete black-outs will result. Utilities can change consumer's habits through the VPP concept and provided bonuses as rewards for customers. Another option is to manage the SG entities through alternative technological paradigms, for instance the energy Internet (EI) paradigm or the software defined networking (SDN) paradigm. The EI paradigm can manage energy transmission and energy dispatching. In particular, the EI paradigm can be used to connect devices together in the SG and to manage power flows among different entities [16]. The use of the SDN paradigm in the SG is outlined as a future research direction in Section IV-C. However, in our opinion, the EI and SDN paradigms are general paradigms that can be adapted to accommodate heterogeneous RERs in the SG. In contrast, the VPP concept is a cohesive solution that has been specifically designed to manage heterogeneous entities, such as RERs, in the SG. Thus, we mainly consider the VPP concept in this paper.

\section{INTEGRATION OF RERS WITH THE SG AND COMMUNICATION NETWORKS}

\section{A. Projects Considering Integration of RERs into the SG}

There have been recent advances in the deployment and testing of the SG at the international level. Table I discusses state-of-the-art projects integrating disparate and intermittent RERs into the SG, indicating the project name, the deployment location, the generation capacity, and the type of employed RERs.

For instance, the study [3] has presented the project implemented in the Bear Mountain Wind (BMW) Farm in British Columbia, Canada. This study explained the selection process for effective communication systems for supervisory control and data acquisition (SCADA), power protection, and control for the BMW Farm. Similarly, the British Columbia Institute of Technology (BCIT) has established a campus based microgrid powered by RERs. The study [17] has examined the performance of the ZigBee and WiMAX based communication network in the BCIT microgrid. BCIT is the first campuswide microgrid, located on the BCIT campus in Vancouver. The microgrid is composed of several small generation plants (including PV modules and wind turbines, as well as a thermal turbine and a Nebraska boiler), loads on the campus (including offices, classrooms, welding shop, and residences), and a meshed network. The microgrid has two control entities: a microgrid controller, which is responsible for the supervisory actions in the microgrid, and a microgrid network control center, which is responsible for managing applications, such as demand response, and load shedding. From the communication perspective, the BCIT microgrid consists of three networks, namely the home area network (HAN), the local area network (LAN), and the wide area network (WAN). ZigBee is used in the HAN, narrowband power line communication (NB PLC) is used in the LAN, and WiMAX is used in the WAN.

At the WAN level, reliable packet delivery is critical for the proper operation of the BCIT microgrid. However, the evaluations in [17] found that the packet loss is up to $4.6 \%$ for small 64 Byte packets. This high packet loss occurred due to the Ethernet stack of the radio controller interface of the WiMAX module and this module cannot be optimized without consultation with the WiMAX equipment manufacturer. Thus, the evaluation study in [17] has demonstrated that adopting WiMAX for WAN communication in microgrids is not suitable until the WiMAX WAN communication has been optimized for the communication requirements of the microgrid. Based on this insight, the research study [17] proceeded to optimize the WiMAX settings in close cooperation with the WiMAX equipment manufacturer in order to optimize the WiMAX communication for the considered microgrid settings. This optimization is necessary because WiMAX served as the backbone communication medium for BCIT substation communication and such type of communication requires $100 \%$ reliable packet delivery for small packet (upto 64 Bytes). This optimization by the WiMAX equipment manufacturer 
TABLE I

OVERVIEW OF PROJECTS INTEGRATING DISPARATE AND INTERMITTENT RENEWABLE ENERGY RESOURCES (RERS) INTO THE SG (SECTION III-A).

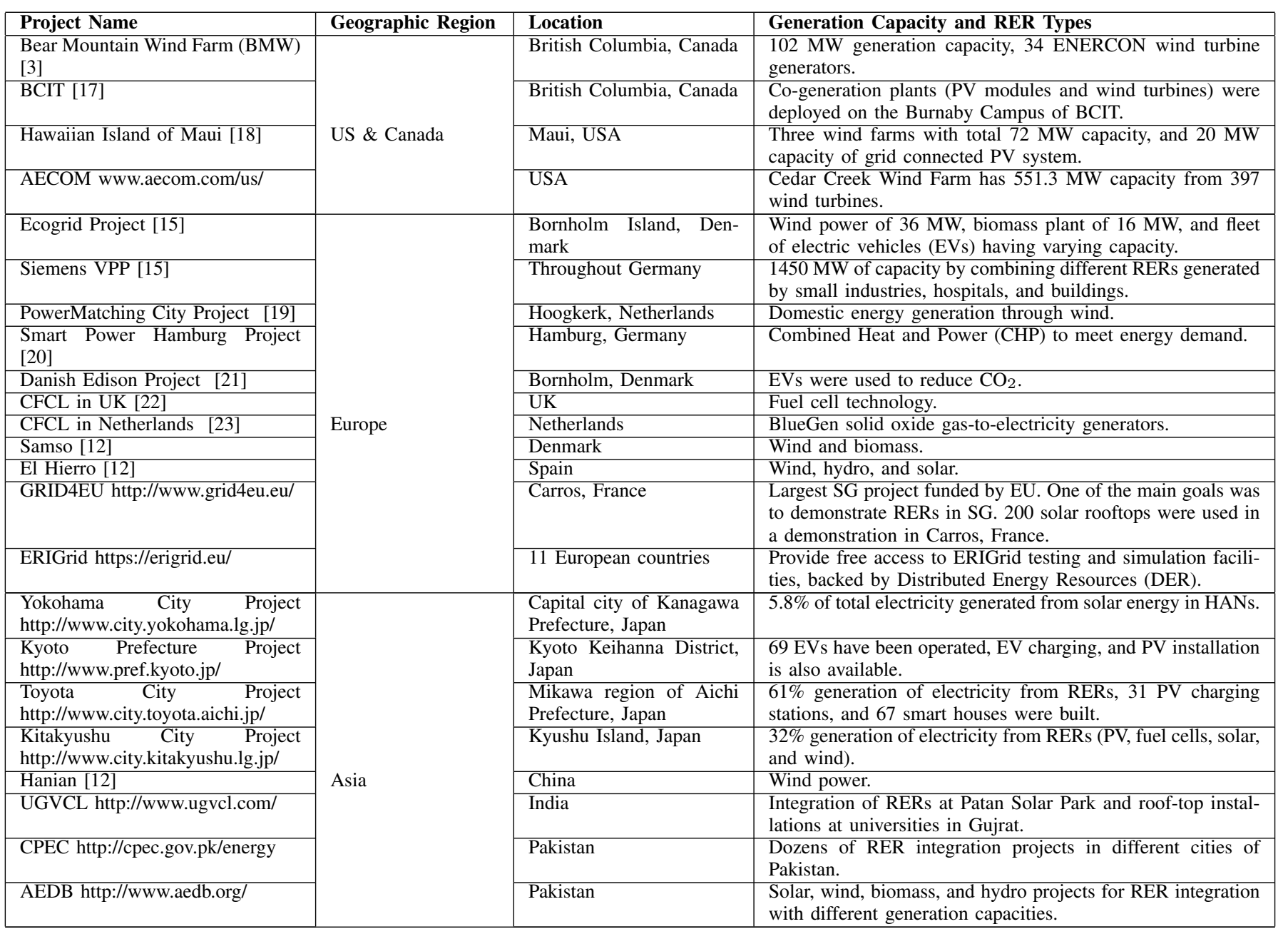

for small packets resulted in the decreased performance for full size packets. Therefore, such modifications require the need to look fundamentally for the design of the protocols which not only performs well for small size packets but also provide optimal performance for full-size packets. It is important to note here that for a practical network deployment and operation scenario it would be prohibitive to collaborate with the WiMAX equipment manufacturers to optimize the WiMAX settings. Thus, there is a need to design wireless communication protocols specifically for the SG communication needs.

At the LAN level, the study [17] adopted ZigBee networks, which were deployed in the Northern part of the BCIT campus and the residential areas. However, the study found that the ZigBee networks were receiving severe interference from WiFi networks, if the ZigBee networks were co-located with WiFi networks, in the same residential area, as both technologies operate on the ISM band. Through extensive experiments, the study [17] concluded that ZigBee networks should select channel 20 to avoid the harmful interference from $\mathrm{WiFi}$ networks. Channel 20 lies in between the WiFi channels. More specifically, channel 20 is located at $2450 \mathrm{MHz}$ which is reserved as a $\mathrm{WiFi}$ channel guard band, i.e., as a frequency band that is intentionally left vacant in between consecutive WiFi channels so as to minimize inter-channel interference. However, forcing ZigBee to statically always select channel 20 is not feasible as ZigBee is based on automatic channel assignment.

These two examples illustrate that the current state-ofthe-art communication technologies are not advanced enough that one could merely pick an appropriate communication technology, tweak it a little bit, and solve the integration issues. Rather, fundamentally novel approaches to the design and optimization of communication network architectures and protocols are required to provide the communications and information technology support needed for the efficient integration of RERs into the SG.

The Hawaiian Island of Maui, US, has abundant RERs, e.g., as wind power. Sometimes, the wind power is so abundant that electricity generation from wind needs to be curtailed as it would overwhelm the power grid. This situations has been investigated in the study [18], which summarized the results of integrating RERs into the power grid. The study [18] also investigated the synergistic effects of EV penetration, RER integration, and methods to store generated renewable energy.

The China Pakistan Economic Corridor (CPEC) has started 
several RERs based energy projects in Pakistan. For instance, a wind farm of $50 \mathrm{MW}$ capacity has been installed in Gharo, Thatta, and two wind farms of $100 \mathrm{MW}$ and $50 \mathrm{MW}$ capacity, respectively, were installed in Jhimpir, Thatta. Similar efforts to promote the use of RERs in Pakistan were also promoted by alternative energy development board (AEDB).

On the Danish island Bornholm, the Ecogrid Project [15] considers multiple RERs, namely wind power, biomass, and EVs. The Bornholm island has a $36 \mathrm{MW}$ wind power plant, a $16 \mathrm{MW}$ of biomass plant, a $2 \mathrm{MW}$ biogas plant, and $5 \mathrm{MW}$ of solar capacity. Gateways were installed on houses and based on energy consumption and prices, appliances in houses can be switched off to conserve energy and sell it to the other consumers that are in need of energy. One of the main findings of the Ecogrid project was that buildings also took part in stabilizing the grid by acting as prosumers, i.e., acting both as producers and consumers of electric energy.

Moreover, in the Danish Edison project, a large range of research and experimental activities were conducted on the island of Bornholm to evaluate how a large fleet of EVs can help in the grid operation as well as provide benefits to the EV car owners [21]. In the study [21], the focus was on the ICT aspects, i.e., how to efficiently integrate the distributed software in the deployed system. The study [21] proposed a VPP architecture referred to as Edison electric vehicle virtual power plant (EVPP). Through the EVPP, the goal was to use fleets of EVs to balance the energy supply provided by variable wind energy resources.

Overall, Denmark has been working hard to use RERs. Another example is the effort to make Samso island (located at Kattegat, Denmark), to completely rely on RERs [12]. Onshore wind turbines of $11 \mathrm{MW}$ capacity and off-shore wind turbines of $22.5 \mathrm{MW}$ capacity were installed to meet the energy requirements of the island. The ultimate goal of the island is to achieve $100 \%$ reliance on renewable energy. That is, even the transportation system should rely on renewable energy and not consume any conventional fossil fuels. To achieve this, the Samso island administration has promoted the use of electric vehicles. Furthermore, the use of biogas from waste for the production of electricity and heat has been one of the top priority of the island's administration.

The PowerMatching City Project [19] in Hoogkerk, Netherlands, has examined the power imbalance effects of intermittent RERs (in particular, wind turbines) on the power grid. In the PowerMatching project, a VPP is responsible for coordinating and managing the decentralized generation and the demand response within the houses. Clusters of houses are created to shift energy generation and consumption within the cluster in order to mitigate the imbalance introduced by the wind turbines.

The Smart Power Hamburg (SPH) project [20] has been deployed in the city of Hamburg, Germany, to achieve improvement gains in combined heat and power (CHP) systems. The goal of SPH was to demonstrate the use of the virtual power plant (VPP) concept for the increasing the heat storage by integrating urban infrastructure heating storage capacity, such as swimming pools, bunkers, and heating networks. In this context, a VPP was developed which helps coordinate the management of RERs in Hamburg.

The existing studies examining the integration of RERs with the SG and communication networks are summarized in Table II. We proceed to survey in detail the integration studies, which are also summarized in Table II.

\section{B. Integration of RERs in Rail Transport System}

Electricity based rail transport is one way to provide safe transportation services to urban and non-urban commuters. In these transportation systems, communication based train control (CBTC) provides safe operation of rail vehicles compared to traditional control systems. Generally in electric vehicles, regenerative braking is used. Regenerative braking is a good source of green renewable energy in which kinetic energy generated by rail transport systems is converted into electrical energy and injected back to the power grid. CBTC combines control and communication technologies which rely on the timely exchange of information. More precisely, accurate and timely sharing of information between trains and wayside equipment to create control commands is vital for the optimal performance of CBTC. Generally, WLANs are used in CBTC systems which are prone to high delays and packet losses due to the mobility of trains. CBTC in conjunction with RERs can be a viable solution to adopt by the train operators to reduce their OPEX and to also reduce $\mathrm{CO}_{2}$ emissions [24]. More precisely, by using reinforcement learning and Q-learning techniques, the study [24] analyzed the impact of introducing CBTC with regenerative braking into the SG. The proposed CBTC scheme has been validated through practical field tests in the Beijing Yizhuang Subway line.

Similarly, the study [25] exploited energy from regenerative braking as a potential RER to provide power charging services to EVs in railway station parking lots. The study [25] proposed to charge EVs through this regenerative renewable energy during the day in order to achieve environmental and economic gains. The proposed approach has been evaluated based on the rail data provided by the Spanish state owned railway infrastructure management company ADIF. However, a limitation of the study [25] is that it is written in Spanish and not easily readable by the general English readers around the globe.

The use of hybrid RERs, such as regenerative energy, solar power, and wind power, for the optimal operation of electric railway systems has been proposed in [26]. The proposed methodology has been validated through a case study based on the Spanish electric railway system. The case study in [26] has demonstrated that substantial cost and energy savings can be achieved through the integration of RERs.

The regenerative braking energy from metro trains in urban transportation systems can be used as an RER for powering other metro trains or even EVs. The study [27] has conducted a case study for this use of the regenerative braking energy of metro trains for the metro system of Madrid, Spain. The goal of this study was to evaluate the effectiveness of using RERs for EVs and metro trains. The study [27] concluded that the use of regenerative braking energy from metro train can reduce the costs for operating the metro system while using EVs as energy storage devices. 


\section{Integration of RERs in Data Centers and WDM}

With the emergence of new Internet services such as big data analytics, mobile cloud computing, and a vast array of cloud based services, the need for data centers is increasing day by day. Social media companies, e.g., Facebook, LinkedIn, Twitter, and YouTube, as well as email service providers, e.g., Gmail and Yahoo, heavily rely on data centers to store and retrieve data. Data centers are designed to support high performance computing applications and they are a major consumer of electricity. Recently, power management in data centers has been addressed by researchers. In data centers, different components are responsible for overall power consumption. More than $80 \%$ of the energy is consumed by servers and cooling equipment [28]. Therefore, with RERs, the performance of data centers as well as the power grid can be optimized [29]. For example, the study [29] considered the data center as an "interruptible" load that can be scaled down when required during peak hours to stabilize the power grid. In order to achieve this scaling-down goal, the study [29] proposed a framework in which the data center is equipped with small software patches that are treated as sensors placed in different sub-systems of the data center. These software patches (sensors) conduct real-time monitoring of the power usage and the ICT activities. The evaluations in [29] employed the Singapore Power (SP) PowerGrid data set and Cloud3DView as a simulation tool.

The study [30] considered Internet Protocol (IP) over Wavelength Division Multiplexing (WDM) networks and showed that by using RERs, the network's $\mathrm{CO}_{2}$ emission can be reduced by an average of $78 \%$. The study [31] exploited the location diversity feature of data centers and distribute the work load among different geographically located data centers based upon available renewable energy resources. Authors, through extensive simulations of real data center data, showed that the cost of running a data center is reduced by $29 \%$ when using their proposed approach. Similarly, the study [32] showed that $71 \%$ power can be saved by using RERs for IP over WDM networks with data centres. In fact, the study [32] optimized the data center locations with respect to the availability of RERs to achieve such high power savings.

The study [33] considered the trade-off between maximizing data center's revenue and minimizing their energy consumption. To investigate this trade-off, authors proposed an optimization based scheme for two different scenarios. In the first scenario, renewable energy is considered, while in the second scenario, renewable energy is not considered. Wind turbines are considered as renewable energy resource in the proposed scheme. The unique feature of this study is that authors considered actual service level agreements (SLA) that exists between customers and data centers. A similar approach to shift the data center's work load is proposed in [34]. However, instead of focusing on SLAs or maximizing the revenue, authors focused on renewable energy future contracts for data centers. Authors then proposed a grouping strategy to cluster those data centers which fall short for their energy requirement or which need to buy more power than estimated.

The study [35] adopted a different way to integrate renew- able energy resources to the data centers. Instead of focusing only on on-site renewable energy resources, authors focused on off-site renewable energy resources such as buying RERs from third party renewable energy providers or buying renewable energy certificates or buying power purchase agreements. Authors concluded that a hybrid of adopting all these options will provide substantial reduction in carbon footprint. Another similar study is conducted in [36] where authors proposed a heuristic approach to identify data centers next to renewable energy resources or to find a way to transmit renewable energy to the data centers. By using the proposed heuristic, $71 \%$ power consumption can be minimized.

A wide area data centers prototype, GreenStar Network (GSN) testbed, powered by renewable energy sources is proposed in [37]. The basic philosophy behind GSN testbed is "follow the wind, follow the sun", i.e., GSN virtually migrates the workload at data centers based upon the availability on renewable energy resources at run time. Experiments were conducted in a scenario containing 13 data centers geographically located across seven cities in the world. In the context of GSN testbed, another study is reported in [38]. Authors basically conducted experiments in GSN data centers and demonstrated that with effective migration of virtual machines nearby renewable energy resources, $\mathrm{CO}_{2}$ emissions can be reduced significantly. The study [39] considers integration of RERs in data centers from the perspective of planning. To be more precise, authors used mixed integer linear programming to determine the location and capacity of the data centers, while considering wind power generation related constraints. Moreover, the study [39] minimized the operation cost of the data center as well as the data routes and the installation cost. For the evaluation purpose, IEEE 118 bus power system was used along with $18 \mathrm{MW}$ wind unit generation capacity.

Recently, the study [40] proposed a planning model for Internet-based large scale data centers based upon different parameters such as availability of on-site RERs, type of microgrid (e.g., AC, DC, or hybrid), and server types along with their capacities. The proposed planning model determines the optimal sites of data centers subject to the considered constraints. One of the unique features of this proposed model is that it captures the short term operations of data centers in the long term planning while considering the variability of RERs. The proposed problem is modelled through a mixed integer linear program and has been solved with the CPLEX optimization software. The results showed that large size renewable energy non-dispatchable units (PVs, and wind units) will be a good choice because of the lower investment cost per MW capacity for large size renewable energy non-dispatchable units (PVs, and wind units) compared to the per MW capacity investment cost for small size non-dispatchable units.

\section{Integration of RERs in Cellular Networks and Internet Services}

Wireless communication is in high demand these days and is provided to the users via wireless access networks. Users are equipped with mobile phones a.k.a., mobile terminals (MTs) to access the base stations (BSs) or access points (APs) in 
TABLE II

OVERVIEW OF STUDIES ON THE INTEGRATION OF RERS INTO COMMUNICATION NETWORKS.

\begin{tabular}{|c|c|c|c|}
\hline Study & Communication Network Type & ICT supporting SG & RER type used \\
\hline$[24]$ & \multirow{4}{*}{ Rail Transport System, Sec. III-B } & $\begin{array}{l}\text { Communication based } \\
\text { train control (CBTC) }\end{array}$ & $\begin{array}{l}\text { Impact of regenerative braking on the SG is evaluated through field } \\
\text { test in Beijing Yizhuang Subway line, China. }\end{array}$ \\
\hline [25] & & Railway traction system & $\begin{array}{l}\text { Regenerative braking as RER is used to charge EVs during the day in } \\
\text { the railway station parking lot. Data from Spanish state owned railway } \\
\text { infrastructure management company ADIF is used for validation. }\end{array}$ \\
\hline [26] & & Electric railway system & $\begin{array}{l}\text { Mix of RERs, i.e., regenerative energy, solar energy, and hybrid energy } \\
\text { is considered. Case study is performed based on the Spanish electric } \\
\text { railway system. }\end{array}$ \\
\hline [27] & & Metro line and EVs & $\begin{array}{l}\text { Metro regenerative braking energy is used to charge EVs. Case study } \\
\text { was conducted based on Madrid metro system. }\end{array}$ \\
\hline [29] & \multirow{12}{*}{ Data Centers and WDM, Sec. III-C } & Data Centers & $\begin{array}{l}\text { Data centers were considered as interruptible load and scaled down to } \\
\text { minimum functionality which may be supported by RERs. }\end{array}$ \\
\hline [30] & & WDM & $20 \mathrm{~kW}, 40 \mathrm{~kW}, 60 \mathrm{~kW}$, and $80 \mathrm{~kW}$ solar power units are considered. \\
\hline [31] & & Data Centers & $\begin{array}{l}20 \text { General Electric } 1.5 \text { Megawatts wind turbines were considered as } \\
\text { behind-the-meter renewable power generator. }\end{array}$ \\
\hline [32] & & WDM with data centers & $\begin{array}{l}\text { Wind farms with } 300 \mathrm{MW}, 400 \mathrm{MW} \text {, and } 700 \mathrm{MW} \text { capacities are con- } \\
\text { sidered. }\end{array}$ \\
\hline [33] & & Data Centers & Each data center is equipped with $1.5 \mathrm{MW}$ wind turbine. \\
\hline [34] & & Cloud Data Centers & Type of renewable energy used is not specified. \\
\hline [35] & & Data Centers & A combination of solar and wind power is considered. \\
\hline [36] & & Data Centers & $\begin{array}{l}\text { Wind farms with } 300 \mathrm{MW}, 400 \mathrm{MW} \text {, and } 700 \mathrm{MW} \text { capacities were } \\
\text { considered along with solar energy. }\end{array}$ \\
\hline [37] & & Data Centers & $\begin{array}{l}\text { Solar, hydro, and wind power is used in the GreenStar Network } \\
\text { testbed. }\end{array}$ \\
\hline [38] & & Data Centers & $\begin{array}{l}\text { Solar, hydro, and wind power are used. Each solar panel has } 220 \\
230 \mathrm{~W} \text { capacity, while the wind turbine has } 15 \mathrm{~kW} \text { generation capacity. }\end{array}$ \\
\hline [39] & & Internet Data Centers & Wind turbine of $18 \mathrm{MW}$ generation capacity is considered. \\
\hline [40] & & Internet Data Centers & Wind and solar energy are considered. \\
\hline [41] & \multirow{5}{*}{$\begin{array}{l}\text { Cellular Netw. \& Internet Services, } \\
\text { Sec. III-D }\end{array}$} & Wireless BS in Smart Grid & Solar power of $300 \mathrm{~W}$ capacity is considered. \\
\hline [42] & & Internet Services & $\begin{array}{l}\text { Study considered prices of green energy, but did not specifically } \\
\text { mentioned the type of green energy used. }\end{array}$ \\
\hline [43] & & BS in Smart Grid & Solar energy panel of $6 \mathrm{~kW}$ capacity is considered. \\
\hline [44] & & BS in Smart Grid & $\begin{array}{l}\text { Solar and wind energy are suggested to use with BSs but generation } \\
\text { capacity values are not mentioned. }\end{array}$ \\
\hline [45] & & Cloud Computing & A 30MW capacity wind power generation unit is considered. \\
\hline
\end{tabular}

order to communicate with other users or with the Internet. It is estimated that there are three billion MTs and three million BSs world wide [46], [47]. Due to the increasing demands for services and applications, such as audio/video streaming, VoIP, and social media applications, offered by wireless communication, the use of MTs and consequently the deployment of BSs will further increase.

Both the MTs and the BSs require energy to operate. This energy is provided by the traditional electrical grid which is generally powered with fossil fuels. Users at home charge their MTs with the electricity, while mobile phone operators feed the electricity from the utilities to their BSs to ensure the smooth operation of their communication networks. The total power consumption of MTs and BSs is $0.2-0.4 \mathrm{GW}$ and 4.5 GW, respectively [46], [47]. This high energy consumption contributes to approximately $2 \%$ of the total $\mathrm{CO}_{2}$ emissions worldwide and this percentage will further grow with the increase of MTs and BSs. This is an alarming situation for both the users and the telecommunication industry, in terms of environmental pollution. Moreover, the operational expenditures (OPEX) for fossil fuel based energy in the telecommunication industry range from $18 \%$ to $32 \%$ [48], [49]. If multiple RERs are used by the telecommunication service providers to power their BSs then both their OPEX and the $\mathrm{CO}_{2}$ emissions can be reduced at global level [43], [44].

BSs based on RERs may also be a good choice for re- mote (off-grid) areas where traditional energy resources are not available, or for developing countries with missing or unreliable traditional energy resources [50]. RERs provide a long-term solution for the stainability of off-grid BSs; however at the expense of capital investments for the renewable energy equipment. BS sleep modes have been investigated for saving energy. Indeed, BSs based on RERs may not be able to support all the traffic and may need to be switched off due to the intermittent nature of RERs. One solution to cope with this situation is to store the renewable energy from RERs and then use it when on-grid energy does not suffice [41], [43], [44]. A detailed discussion on energy-efficient communications and their interaction with the grid is given in [51].

The study [41] focused on adaptive power management of BSs. Through adaptive power management schemes, a BS can communicate with the utility to negotiate and adjust power buying prices according to different criteria, such as traffic load, power price, and generated renewable energy. Through extensive simulations, the study [41] demonstrated that effective adaptive power management can significantly reduce the power cost to operate BSs.

The similar power management study [43] focused not only on reducing the energy cost incurred by the BSs (and ultimately the network operator) but also strived to maintain high levels of Quality of Service to the users. Based on real BS scenarios that are deployed in London, UK, and powered 
through solar energy, the study demonstrated that intelligent power management can significantly reduce the consumed grid energy while maintaining high QoS levels.

Cellular network operators strive to minimize the energy consumption of their overall networks. When BSs are equipped with RERs, it may happen that some BSs have a shortage of renewable energy; while nearby BSs have a surplus of stored renewable energy. Thus, if the surplus renewable energy is exchanged with the BSs that have a shortage, then an overall energy balance can be achieved. An energy sharing framework to achieve this overall energy balance among BSs has been proposed in [44]. The key feature of this framework is that it considers battery capacity and real-time pricing when procuring and exchanging energy among the BSs. Moreover, the framework study in [44] investigated three cases i.e., when renewable energy generation by the BSs is unknown, partially known, and perfectly known ahead of time. Results showed that the energy exchange among BSs achieved significant reductions of the average energy cost.

The study [42] proposed an optimization framework to reduce the brown energy (a.k.a the energy generated from conventional fossil fuels, such as coal or oil) consumption for Internet services, while maintaining the prescribed SLAs. The framework considers the different time zones of data centers for cost minimization and accounts for a wide range of energy parameters, such as varying electricity prices of green and brown energy.

By using cloud computing in conjunction with data transfers through the Internet, weather reports, tidal conditions, and data generated by wind turbines can be easily collected, stored, analyzed, and accessed by the utilities and the customers. The study [45] has presented a cloud-based communication and control framework for the integration of wind energy into the SG. In particular, the presented framework exploits cloud computing in conjunction with effective and reliable communications to create a control system for the integration of wind energy into the SG. Input parameters, such as the specifications and performance data of the generation units and consumption data of the consumers, are communicated to the cloud and the cloud then computes the power allocations for the generation units. The power allocations are then communicated to and implemented by the control system. The study [45] demonstrated that the cloud-based framework can reduce the processing time for control decisions, reduce the communication bandwidth, and achieve resilient control.

\section{Future Research Directions for Integrating RERS INTO THE SG}

There are several challenges that need to be addressed in order to roll out the SG. These challenges may slow down the implementation of the SG or, if they are not properly resolved, they may impede the full utilization and realization of the futuristic SG. These challenges include the proper planning of the integration of RERs into the SG, scalability issues, regulatory issues, the standardization of universal protocols, and standards for the seamless operation of the SG. In this section, we discuss future research directions related to the integration of RERs into the SG.

\section{A. Interoperability of Different Communication Systems}

A major challenge faced by utilities is the lack of interoperability of the different communication systems deployed in different SG segments. In order to address this interoperability challenge, the U.S. National Institute of Standards and Technology (NIST) has been working on an interoperability project, i.e., IEEE P2030 in which interoperability issues related with the integration of power system, energy resources, and information technology will be addressed [3], [52].

ZigBee Smart Energy is an interoperability standard for HANs and has been successfully implemented in BC Hydro (British Columbia), California, and Texas [4]. However, there is a need to further intensify the research and development efforts for protocols and standards that efficiently address the interoperability of different SG communication systems.

\section{B. Lack of Standard Interface}

The VPP aims to provide a conceptual structure to unify the different RERs and SG components. However, a critical open challenge is the development of a standard interface that joins all of these RERs and SG components together. Despite the fact that there have been several ongoing projects on the integration of RERs with the SG (cf. Table I), there is an urgent need to learn from each others experiences while designing new projects. For instance, BCIT's smart microgrid testbed can provide insights to designers of new projects. BCIT had to cope with two main problems during the implementation of a communication system in the microgrid [17]. The first problem was related to the high packet losses at BCIT's WiMAX enabled WAN backbone and the second problem was related to the interference between the deployed ZigBee network and the campus WiFi network. The solutions and insights of the BCIT team to handle these two problems can be helpful for new project designs.

Possibly, the development of the standard VPP interface can take lessons from the recent development of the OpenFlow interface [53] between controllers and switches in the context of the software defined networking (SDN) paradigm for communication networks. Similar to the way that standard interfaces facilitated virtual machines in the computing field since the 1960s, the standard OpenFlow interface and SDN paradigm are facilitating network virtualization [54], i.e., the flexible aggregation and slicing of network resources [55][57]. VPPs could similarly aggregate and slice RERs through a uniform standard interface definition.

\section{Software Defined Networking (SDN) based Smart Grid}

More generally, the SDN paradigm with its capability to separate the control plane from the data plane, can be broadly used as a basis for SG control support. SDN has been proposed to monitor and manage the communication networks globally. Since the SG relies heavily on communication networks for control, SDN can be employed to manage the communication entities in the SG system and then be extended to form a basis for SG control. By applying SDN in SG systems, efficiency and resiliency can potentially be improved. For instance, the 
SDN based SG can be used for load balancing and shifting, for dynamically adjusting the routing paths for SG control commands, and for monitoring and scheduling of critical SG traffic flows. However, separating the control plane (using a single controller), may endanger the critical SG infrastructure and may create a single point of failure that is prone to Denial of Service (DoS) attacks. Therefore, the use of SDN as a basis for SG control needs to be investigated in detail.

A microgrid is essentially an independent power grid using RERs to serve customers within its jurisdiction. For instance, a university campus or a commercial building can be served as a microgrid. Considering the energy requirements of customers within a microgrid, it may happen that one microgrid has an energy shortfall which may be compensated for by borrowing surplus energy from a neighboring microgrid, thus forming a network of microgrids, i.e., so-called networked microgrids (NMGs). This energy exchange between NMGs requires timely reliable inter-NMG communication, i.e., a reliable inter-NMG communication system. SDN can help to create and control this inter-NMG communication. However, there is a need to develop application programming interfaces (APIs) for the east bound interface (EBI) to network domains not operating SDN and the west bound interface (WBI) to network domains operating SDN. In particular, a west bound interface will help the microgrid to communicate with other SDN based NMGs and an east bound interface will help the microgrid to communicate with SG communication infrastructures employing legacy protocols, such as multi protocol label switching (MPLS).

Software Defined Optical Networks (SDONs) [53] are another future research direction where optical networks are used for reliable communication in the SG system. More precisely, in a substation communication network, a hybrid opto-electric Ethernet can be placed for control message communication using the IEC 61850 protocol. However, in SDONs, both the control and the infrastructure depend on physical fiber which requires proper management schemes.

\section{Smart Grid Infrastructure as a Service}

The SG will be composed of a diverse range power and communication infrastructures, electronic equipment pieces, and devices, ranging from smart meters to Phasor Measurement Units (PMUs), and from wind turbines to thousands of mobile Plug-in Electric Vehicles (PEVs). By using the concept of virtualization together with SDN, these electronic devices and SG infrastructures, can be shared among multiple parties (e.g., utilities and customers) and each party will be given full control over its slice of the resources. This development can lead to the emergence of a virtual sub-station concept. By doing so, the concept of smart grid infrastructure as a service (SG-IaaS) can be developed. For instance, a utility can combine geographically dispersed RERs and create a virtual sub-station. However, business and revenue models for this SG-IaaS concept need to be extensively studied. In the context of 5G networks, the network slicing paradigm consists in dividing the network into isolated network slices which enables the power/smart grid to be programmable and customizable to different vertical industries need. One network slice can be dedicated to RER applications to make efficient, secure and with high-quality smart grid services.

\section{E. Network Coding in Smart Grid}

Network coding brings numerous advantages, such as improved reliability and reduced delay. Given these advantages of network coding, the application of network coding in the SG context is a highly promising direction for future research [58]. More precisely, an SG based on RERs generates different types of traffic with diverse QoS requirements in terms of reliability, delay, and throughput. For instance, wide area situational awareness traffic in the SG requires $99.99 \%$ reliability and 20 $200 \mathrm{~ms}$ delay. Another SG traffic scenario is the bulk transfer of meter readings from the Advanced Metering Infrastructure (AMI) to the utility. This bulk transfer traffic type is not delay sensitive, but may require the transfer of large data amounts on the order of Mega bytes. Network coding can be used in the SG to increase the throughput, to enhance the reliability, and to decrease the number of transmissions by re-coding data while it is in transit from the AMI to the utility. Similarly, the speed of data gathering from different SG entities can be increased with network coding.

Future research on network coding in the context of the SG needs to thoroughly investigate the transport of these SG traffic types. Moreover, there is generally a need to thoroughly investigate the integration of network coding protocols with the SG communications architecture and protocols to efficiently transport the SG traffic flow data generated from diverse devices and applications. Important SG traffic flow transport challenges include the orchestration of traffic flows, the management of traffic flow prioritization, and the provisioning of a wide range of QoS levels from relaxed to stringent QoS requirements.

\section{F. Comprehensive Evaluation Framework}

SG systems are complex made up of different sub-systems, including the electrical, control, and communication subsystems. In addition, since the future SG will likely be an inverter-dominated grid, RERs will be tightly coupled with the SG system. RERs are typically intermittent in nature, are based on highly heterogeneous technologies, and are typically spread out in terms of their geographic locations; therefore, the simulation and validation of these systems is highly complex. An important future research direction is the development of co-simulation frameworks and testbeds that rigorously examine and validate the performance of SG systems in the presence of variable heterogeneous distributed RERs. Co-simulation approaches will incur communication delays between different SG sub-systems, which will need to be carefully accounted for. Moreover, a comprehensive evaluation framework for a systematic evaluation and validation of the suite of protocols for the full range of SG operating conditions needs to be developed. 


\section{G. Integration of RERs into Data Centers}

RERs can also be integrated with data centers to optimize their performance. One direction is to schedule different component "electrical loads" of data centers on different energy resources or an energy mix can be used to perform tasks [29]. Moreover, cooling is a major issue in data centers, therefore, batch jobs can be executed at night with outside air cooling at lower prices, while regular jobs can be executed when solar energy is available during daylight hours. In this context, scheduling schemes need to be designed that consider the availability characteristics of the different RERs [28].

One way to reduce $\mathrm{CO}_{2}$ emissions is to dynamically shift the load of data centers that are powered by RERs (cf. Sec III-C). The effectiveness of this approach has been demonstrated in the GSN data center case study [37]. However, the generalization of this strategy to large-scale and mega-scale data centers, such as the Google or Microsoft data centers is an open problem. One way to tackle this open problem could be to physically locate the data centers in different geographical locations, each powered by RERs [38].

\section{H. Demand Side Management, Load Shifting, and Dynamic Energy Pricing at Data Centers through RERs}

With the incorporation of RERs into data centers, the load can be dynamically shifted from RERs to the main power grid and vice versa. Since data centers consume enormous amounts of energy (e.g., typically 91 billion kilowatt-hours [28] annually), they may help to stabilize the powers grid by temporarily shifting to RERs (data centers may also scaledown to minimum functionality which may be supported by RERs).

Another challenge is to design techniques to dynamically procure energy from utilities and to optimize energy prices in order to earn profits while operating based on RERs.

\section{Anti-Islanding Techniques and Communication Systems}

With the incorporation of more DERs and RERs into the $\mathrm{SG}$, there are increasing risks that parts of the grid become isolated during faults. Besides requiring anti-islanding techniques, communication protocols will be required to communicate such events to the control center so as to mitigate islanding faults [2], [17]. Moreover, novel disconnection tolerant networks (DTNs) and opportunistic networks would be useful for future communications for anti-islanding techniques and communication systems [59].

\section{J. Cognitive Radio Supporting RER Communication in SG}

A typical SG will consists of thousands of smart meters deployed at user premises. These smart meters will be required to communicate with local access points to send or receive information from the utilities. Smart meters use wireless spectrum bands (e.g., the $2.4 \mathrm{GHz}$ ISM band) and may cause interference with other wireless networks operating in the vicinity. Smart meters may use cognitive radio (CR) technology for interference-free wireless communication [60].
Generally, the different SG entities need to communicate, requiring wireless spectrum bands if wireless communication is employed. CR can be a good candidate for mitigating the spectrum scarcity problem by exploiting spectrum white space. Integrating RERs into the SG will heavily dependent on flexible reliable communication among the different SG components, which can be efficiently supported with CR communication [60].

\section{K. Heterogeneous Applications and Friendly User Interfaces}

Consumers and utilities can reap the full benefits of RERs integrated with SG systems when useful applications with user-friendly front-ends and GUIs will be developed. These applications will collect data from various sources in the SG system and display them to the users. A user friendly GUI will not only enable the consumer to monitor the energy consumption, but it will also help the consumer to compare the consumed energy cost with historical data. In addition, the GUI will enable the consumer to control appliances remotely, to shift electric loads to and from RERs, to switch appliances on and off, and to monitor the appliances. However, the Quality of Experience (QoE) needs to be carefully considered while designing such applications. For instance, in a smart home energy management system equipped with RERs, appliances can be scheduled (e.g., starting time of a washing machine can be deferred by a few hours subject to the availability of RERs) to reduce the electricity cost, but consumer preferences and any inconveniences caused for the consumer need to be considered. This can be achieved by developing QoE aware applications that consider the input from the consumer to adapt scheduling decisions in the future while taken into account the tradeoffs between the consumer preferences and the electricity cost.

\section{Big Data, RERs, and Smart Grid}

Geographically distributed RERs generate huge amounts of data. For instance, the already deployed wind farms and solar farms generate extensive data amounts at regular intervals. The efficiency of these wind and solar farms can be further improved with the help of modern big data analytic tools. Similarly, the wind turbine placement can be improved by analyzing weather reports, tidal conditions, and satellites images through big data technologies. Thus, big data analytics may help exploit the enormous data generated by RERs in the SG for optimizations. Future research needs to thoroughly investigate the optimization opportunities. Also, future research needs to develop novel systems that can efficiently collect, process, and exploit the data generated from these sources and integrate the data with the ICT infrastructure in the SG for real-time processing.

\section{Security and Privacy for Integration of RERs into SG}

On one hand, it is estimated that over $50 \%$ of the electricity is consumed in homes [4], while on the other hand, homes are the places where RERs are deployed at a massive level. In-house RERs, such as solar panels and even small scale 
distributed wind farms which are connected with smart homes, can inject back substantial amounts of energy into the grid. Moreover, consumers can relay a wide range of functions, such as demand side management, real time pricing, load scheduling, and software upgrades, through gateways to a number of smart meters without the need to visit every meter location. Information exchanges can occur in the form of meter readings from meters to the utility, from meters to the AMI, and from the AMI to the utility. These information exchanges are either on-demand, scheduled a priori, or in the form of bulk transfers. Real-time pricing and time of use (TOU) pricing information exchanges also occur between the utility and the meters. These information exchanges need to be conducted in a secure manner. Moreover, consumers' privacy should be given top priority while designing information exchange algorithms and protocols.

\section{CONCLUSION}

With the help of information and communication technologies, advanced control systems, and sensing techniques, the current electricity grid is being upgraded to the so-called smart grid (SG). One of the unique SG features is the incorporation of renewable energy resources (RERs). RERs not only facilitate the injection of surplus energy from small consumers back into the grid, but also help to reduce $\mathrm{CO}_{2}$ emissions. In this paper, we have discussed RERs and the issues arising from integrating RERs into the SG, with a focus on different types of communication networks. We have highlighted different ongoing projects around the globe that integrate disparate and intermittent RERs into the SG. We have observed that communication systems play a vital role in the integration of RERs into the SG. Without these communication systems, two-way communication, which is an integral part of the future $\mathrm{SG}$, is not possible. We concluded the survey by outlining future research directions for integrating RERs into the SG.

\section{REFERENCES}

[1] H. Farhangi, "The path of the smart grid," IEEE Power and Energy Magazine, vol. 8, no. 1, pp. 18-28, January 2010.

[2] B. Kroposki, B. Johnson, Y. Zhang, V. Gevorgian, P. Denholm, B. M. Hodge, and B. Hannegan, "Achieving a 100\% renewable grid: Operating electric power systems with extremely high levels of variable renewable energy," IEEE Power and Energy Magazine, vol. 15, no. 2, pp. 61-73, March 2017.

[3] F. R. Yu, P. Zhang, W. Xiao, and P. Choudhury, "Communication systems for grid integration of renewable energy resources," IEEE Network, vol. 25, no. 5, pp. 22-29, September 2011.

[4] B. Heile, "Smart grids for green communications [industry perspectives]," IEEE Wireless Commun., vol. 17, no. 3, pp. 4-6, June 2010.

[5] D. Pudjianto, C. Ramsay, and G. Strbac, "Virtual power plant and system integration of distributed energy resources," IET Renewable Power Generation, vol. 1, no. 1, pp. 10-16, March 2007.

[6] A. Ahmad, M. H. Rehmani, H. Tembine, O. A. Mohammed, and A. Jamalipour, "IEEE Access Special Section Editorial: Optimization for emerging wireless networks: IoT, $5 \mathrm{G}$, and smart grid communication networks," IEEE Access, vol. 5, pp. 2096-2100, 2017.

[7] M. H. Rehmani, M. E. Kantarci, A. Rachedi, M. Radenkovic, and M. Reisslein, "IEEE Access Special Section Editorial: Smart grids: A hub of interdisciplinary research," IEEE Access, vol. 3, pp. 3114-3118, 2015.

[8] Q.-C. Zhong and T. Hornik, Control of Power Inverters in Renewable Energy and Smart Grid Integration. Wiley-IEEE Press, 2013.

[9] A. Moreno-Munoz, Ed., Large Scale Grid Integration of Renewable Energy Sources. IET, 2015.
[10] F. P. Sioshansi, Ed., Smart Grid: Integrating Renewable, Distributed \& Efficient Energy. Academic Press, 2012.

[11] H. Farhangi, "A road map to integration: Perspectives on smart grid development," IEEE Power and Energy Magazine, vol. 12, no. 3, pp. 52-66, May 2014.

[12] H. Chen, P. Xuan, Y. Wang, K. Tan, and X. Jin, "Key technologies for integration of multitype renewable energy sources - research on multitimeframe robust scheduling/dispatch," IEEE Transactions on Smart Grid, vol. 7, no. 1, pp. 471-480, Jan 2016.

[13] T. Adefarati and R. C. Bansal, "Integration of renewable distributed generators into the distribution system: a review," IET Renewable Power Generation, vol. 10, no. 7, pp. 873-884, 2016.

[14] S. Bu, F. R. Yu, Y. Cai, and X. P. Liu, "When the smart grid meets energy-efficient communications: Green wireless cellular networks powered by the smart grid," IEEE Transactions on Wireless Communications, vol. 11, no. 8, pp. 3014-3024, August 2012.

[15] J. Kumagai, "Virtual power plants, real power," IEEE Spectrum, vol. 49, no. 3, pp. 13-14, March 2012.

[16] R. Wang, J. Wu, Z. Qian, Z. Lin, and X. He, "A graph theory based energy routing algorithm in energy local area network," IEEE Trans. on Industrial Informatics, vol. 13, no. 6, pp. 3275-3285, Dec 2017.

[17] G. Stanciulescu, H. Farhangi, A. Palizban, and N. Stanchev, "Communication technologies for BCIT smart microgrid," in 2012 IEEE PES Innovative Smart Grid Technologies (ISGT), Jan 2012, pp. 1-7.

[18] D. Corbus, M. Kuss, D. Piwko, G. Hinkle, M. Matsuura, M. McNeff, L. Roose, and A. Brooks, "All options on the table: Energy systems integration on the Island of Maui," IEEE Power and Energy Magazine, vol. 11, no. 5, pp. 65-74, Sep. 2013.

[19] B. Roossien, A. van den Noort, R. Kamphuis, F. Bliek, M. Eijgelaar, and J. de Wit, "Balancing wind power fluctuations with a domestic virtual power plant in Europe's first smart grid," in Proc. IEEE Trondheim PowerTech, 2011, pp. 1-5.

[20] P. P. Vuthi, P. Lorenzen, H. Schaefers, S. Raths, S. Krengel, J. Sudeikat, and M. Thomsen, "Smart power Hamburg: A virtual power plant for Hamburg," in Proc. Int. VDE ETG Congress; Die EnergiewendeBlueprints for the New Energy Age, 2015, pp. 1-8.

[21] C. Binding, D. Gantenbein, B. Jansen, O. Sundström, P. B. Andersen, F. Marra, B. Poulsen, and C. Træholt, "Electric vehicle fleet integration in the Danish EDISON project-a virtual power plant on the island of Bornholm," in Proc. IEEE PES General Meeting, 2010, pp. 1-8.

[22] "CFCL BlueGENs for Dutch virtual power plant project, UK grid," Fuel Cells Bulletin, vol. 2013, no. 12, p. 6, Dec. 2013.

[23] "CFCL BlueGen units for virtual power plant project in Netherlands," Fuel Cells Bulletin, vol. 2012, no. 7, p. 3, Jul. 2012.

[24] W. Sun, F. R. Yu, T. Tang, and S. You, "A cognitive control method for cost-efficient CBTC systems with smart grids," IEEE Trans. Intelligent Transportation Systems, vol. 18, no. 3, pp. 568-582, March 2017.

[25] J. C. Hernandez and F. S. Sutil, "Electric vehicle charging stations feeded by renewable: $\mathrm{Pv}$ and train regenerative braking," IEEE Latin America Transactions, vol. 14, no. 7, pp. 3262-3269, July 2016.

[26] J. Aguado, A. J. Sanchez-Racero, and S. de la Torre, "Optimal operation of electric railways with renewable energy and electric storage systems," IEEE Transactions on Smart Grid, vol. PP, no. 99, pp. 1-1, 2017.

[27] C. F. Calvillo, A. Sanchez-Miralles, and J. Villar, "Synergies of electric urban transport systems and distributed energy resources in smart cities," IEEE Trans. on Intelligent Transportation Systems, in print, 2018.

[28] W. Zhang, Y. Wen, Y. W. Wong, K. C. Toh, and C. H. Chen, "Towards joint optimization over ICT and cooling systems in data centre: A survey," IEEE Communications Surveys \& Tutorials, vol. 18, no. 3, pp. 1596-1616, Third Qu. 2016.

[29] W. Xia, Y. Wen, K. C. Toh, and Y. W. Wong, "Toward green data centers as an interruptible load for grid stabilization in Singapore," IEEE Commun. Mag., vol. 53, no. 11, pp. 192-198, November 2015.

[30] X. Dong, T. El-Gorashi, and J. M. H. Elmirghani, "IP over WDM networks employing renewable energy sources," IEEE/OSA Journal of Lightwave Technology, vol. 29, no. 1, pp. 3-14, Jan. 2011.

[31] M. Ghamkhari and H. Mohsenian-Rad, "Optimal integration of renewable energy resources in data centers with behind-the-meter renewable generator," in IEEE Int. Conf. on Commun., June 2012, pp. 3340-3344.

[32] X. Dong, T. El-Gorashi, and J. M. H. Elmirghani, "Energy-efficient IP over WDM networks with data centres," in Proc. Int. Conf. on Transparent Optical Networks, June 2011, pp. 1-8.

[33] M. Ghamkhari and H. Mohsenian-Rad, "Energy and performance management of green data centers: A profit maximization approach," IEEE Transactions on Smart Grid, vol. 4, no. 2, pp. 1017-1025, June 2013. 
[34] M. Erol-Kantarci and H. T. Mouftah, "Overlay energy circle formation for cloud data centers with renewable energy futures contracts," in Proc. IEEE Symp. on Computers and Commun. (ISCC), June 2014, pp. 1-6.

[35] C. Ren, D. Wang, B. Urgaonkar, and A. Sivasubramaniam, "Carbonaware energy capacity planning for datacenters," in Proc. IEEE Int. Symp. on Modeling, Analysis and Simulation of Computer and Telecommun. Systems, Aug 2012, pp. 391-400.

[36] X. Dong, T. El-Gorashi, and J. M. H. Elmirghani, "Green IP over WDM networks: Solar and wind renewable sources and data centres," in Proc. IEEE Globecom, Dec 2011, pp. 1-6.

[37] K. K. Nguyen, M. Cheriet, M. Lemay, M. Savoie, and B. Ho, "Powering a data center network via renewable energy: A green testbed," IEEE Internet Computing, vol. 17, no. 1, pp. 40-49, Jan 2013.

[38] M. Lemay, K. K. Nguyen, B. S. Arnaud, and M. Cheriet, "Toward a zero-carbon network: Converging cloud computing and network virtualization," IEEE Internet Comp., vol. 16, no. 6, pp. 51-59, Nov 2012.

[39] A. Vafamehr, M. E. Khodayar, S. D. Manshadi, I. Ahmad, and J. Lin, "A framework for expansion planning of data centers in electricity and data networks under uncertainty," IEEE Trans. on Smart Grid, in print, 2018.

[40] W. Qi, J. Li, Y. Liu, and C. Liu, "Planning of distributed Internet data center microgrids," IEEE Trans. on Smart Grid, in print, 2018.

[41] D. Niyato, X. Lu, and P. Wang, "Adaptive power management for wireless base stations in a smart grid environment," IEEE Wireless Communications, vol. 19, no. 6, pp. 44-51, December 2012.

[42] K. Le, R. Bianchini, T. D. Nguyen, O. Bilgir, and M. Martonosi, "Capping the brown energy consumption of Internet services at low cost," in Proc. Int. Conf. on Green Computing, Aug 2010, pp. 3-14.

[43] V. Chamola, B. Sikdar, and B. Krishnamachari, "Delay aware resource management for grid energy savings in green cellular base stations with hybrid power supplies," IEEE Transactions on Communications, vol. 65, no. 3, pp. 1092-1104, March 2017

[44] M. J. Farooq, H. Ghazzai, A. Kadri, H. ElSawy, and M. S. Alouini, "A hybrid energy sharing framework for green cellular networks," IEEE Transactions on Communications, vol. 65, no. 2, pp. 918-934, Feb 2017.

[45] M. Rayati and A. Ranjbar, "Resilient transactive control for systems with high wind penetration based on cloud computing," IEEE Trans. on Industrial Informatics, vol. 14, no. 3, pp. 1286-1296, Mar. 2018.

[46] S. Mclaughlin, P. M. Grant, J. S. Thompson, H. Haas, D. I. Laurenson, C. Khirallah, Y. Hou, and R. Wang, "Techniques for improving cellular radio base station energy efficiency," IEEE Wireless Communications, vol. 18, no. 5, pp. 10-17, October 2011.

[47] M. Ismail, W. Zhuang, E. Serpedin, and K. Qaraqe, "A survey on green mobile networking: From the perspectives of network operators and mobile users," IEEE Communications Surveys \& Tutorials, vol. 17, no. 3 , pp. 1535-1556, Third Qu. 2015.

[48] T. Chen, Y. Yang, H. Zhang, H. Kim, and K. Horneman, "Network energy saving technologies for green wireless access networks," IEEE Wireless Communications, vol. 18, no. 5, pp. 30-38, October 2011.

[49] C. Han, T. Harrold, S. Armour, I. Krikidis, S. Videv, P. M. Grant, H. Haas, J. S. Thompson, I. Ku, C. X. Wang, T. A. Le, M. R. Nakhai, J. Zhang, and L. Hanzo, "Green radio: radio techniques to enable energyefficient wireless networks," IEEE Communications Magazine, vol. 49, no. 6 , pp. 46-54, June 2011.

[50] J. Wu, Y. Zhang, M. Zukerman, and E. K. N. Yung, "Energy-efficient base-stations sleep-mode techniques in green cellular networks: A survey," IEEE Communications Surveys \& Tutorials, vol. 17, no. 2, pp. 803-826, Second Qu. 2015.

[51] M. Erol-Kantarci and H. Mouftah, "Energy-efficient information and communication infrastructures in the smart grid: A survey on interactions and open issues," IEEE Communications Surveys \& Tutorials, vol. 17, no. 1, pp. 179-197, First Qu. 2015.

[52] "IEEE P2030 draft guide for smart grid interoperability of energy technology and information technology operation with the electric power system (EPS), and end-use applications and loads," Dec 2010.

[53] A. S. Thyagaturu, A. Mercian, M. P. McGarry, M. Reisslein, and W. Kellerer, "Software defined optical networks (SDONs): A comprehensive survey," IEEE Communications Surveys \& Tutorials, vol. 18, no. 4, pp. 2738-2786, Fourth Qu. 2016.

[54] R. Mijumbi, J. Serrat, J.-L. Gorricho, N. Bouten, F. De Turck, and R. Boutaba, "Network function virtualization: State-of-the-art and research challenges," IEEE Communications Surveys \& Tutorials, vol. 18, no. 1, pp. 236-262, First Qu. 2016.

[55] A. Blenk, A. Basta, M. Reisslein, and W. Kellerer, "Survey on network virtualization hypervisors for software defined networking," IEEE Commun. Surveys \& Tutorials, vol. 18, no. 1, pp. 655-685, First Qu. 2016.
[56] A. Blenk, A. Basta, J. Zerwas, M. Reisslein, and W. Kellerer, "Control plane latency with SDN network hypervisors: The cost of virtualization," IEEE Trans. Network and Service Management, vol. 13, no. 3, pp. 366380, Sep. 2016.

[57] A. S. Thyagaturu, Y. Dashti, and M. Reisslein, "SDN-based smart gateways (Sm-GWs) for multi-operator small cell network management," IEEE Trans. on Network and Service Management, vol. 13, no. 4, pp. 740-753, Dec. 2016.

[58] R. Prior, D. E. Lucani, Y. Phulpin, M. Nistor, and J. Barros, "Network coding protocols for smart grid communications," IEEE Transactions on Smart Grid, vol. 5, no. 3, pp. 1523-1531, May 2014.

[59] M. Radenkovic and A. Grundy, "Efficient and adaptive congestion control for heterogeneous delay-tolerant networks," Ad Hoc Networks, vol. 10, no. 7, pp. $1322-1345,2012$.

[60] A. A. Khan, M. H. Rehmani, and M. Reisslein, "Cognitive radio for smart grids: Survey of architectures, spectrum sensing mechanisms, and networking protocols," IEEE Communications Surveys \& Tutorials, vol. 18, no. 1, pp. 860-898, First Qu. 2016.

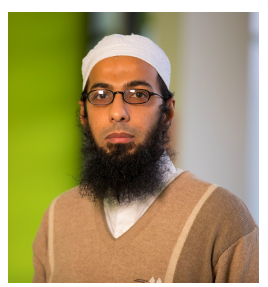

Mubashir Husain Rehmani (M'14-SM'15) is a Post-Doctoral Researcher at Waterford Institute of Technology, Ireland. He received the Ph.D. degree from the University Pierre and Marie Curie, Paris, France in 2011. He currently serves as Area Editor for the IEEE Communications Surveys \& Tutorials and was recognized as "Exemplary Editor of the IEEE Communications Surveys and Tutorials for the year 2015" by the IEEE Communications Society.

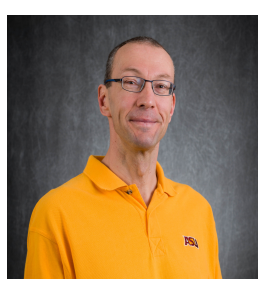

Martin Reisslein (S'96-A'97-M'98-SM'03-F'14) is a Professor in the School of Electrical, Computer, and Energy Engineering at Arizona State University (ASU), Tempe. He received the Ph.D. in systems engineering from the University of Pennsylvania in 1998. He is Associate Editor-in-Chief for the IEEE Communications Surveys \& Tutorials and chairs the steering committee of the IEEE Transactions on Multimedia.

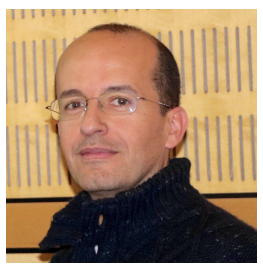

Abderrezak Rachedi (S'05-M'08-SM'15) received the Ph.D. degree in computer science from the University of Avignon, France, in 2008, and the H.D.R. degree in computer science from Paris-Est University, France, in 2015. He has been a member of the Gaspard Monge Computer Science Laboratory since 2008. He is currently an Associate Professor (ma $\tilde{A} 囚$ tre de conferences) with the University ParisEst Marne-la-Vallée (UPEM), France.

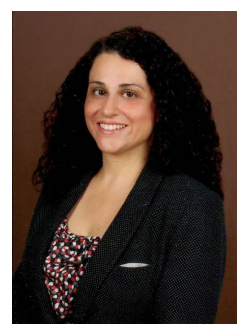

Melike Erol-Kantarci (SM'15) is Assistant Professor at the School of Electrical Engineering and Computer Science at the University of Ottawa. She is the founding director of the Networked Systems and Communications Research (NETCORE) laboratory. She received her Ph.D. and M.Sc. degrees in Computer Engineering, and B.Sc. degree in Control and Computer Engineering from Istanbul Technical University in 2009, 2004 and 2001, respectively.

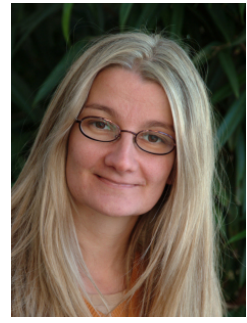

Milena Radenkovic received the Dipl.-Ing. degree in Electric and Electronic Engineering from the University of Nis, Serbia, and the Ph.D. degree in Computer Science from the University of Nottingham, U.K. Her research interests span areas of intelligent mobile and disconnection tolerant networking, complex temporal graphs, self organised security and distributed predictive analytics with applications to autonomous vehicles, mobile social networks, smart manufacturing and telemetry. 